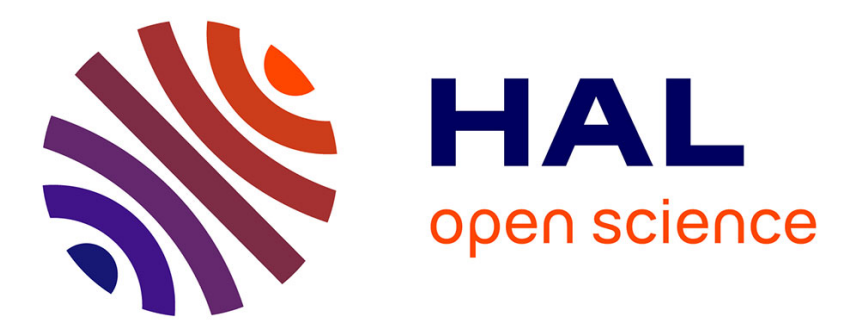

\title{
The Impact of Community Organization on Vector-Borne Pathogens
}

Benjamin Roche, Pejman Rohani, Andy Dobson, Jean-François Guégan

\section{To cite this version:}

Benjamin Roche, Pejman Rohani, Andy Dobson, Jean-François Guégan. The Impact of Community Organization on Vector-Borne Pathogens. American Naturalist, 2013, 181 (1), pp.1-11. 10.1086/668591. hal-02391503

\section{HAL Id: hal-02391503 \\ https://hal.umontpellier.fr/hal-02391503}

Submitted on 3 Dec 2019

HAL is a multi-disciplinary open access archive for the deposit and dissemination of scientific research documents, whether they are published or not. The documents may come from teaching and research institutions in France or abroad, or from public or private research centers.
L'archive ouverte pluridisciplinaire $\mathbf{H A L}$, est destinée au dépôt et à la diffusion de documents scientifiques de niveau recherche, publiés ou non, émanant des établissements d'enseignement et de recherche français ou étrangers, des laboratoires publics ou privés. 


\section{CHICAGO JOURNALS}

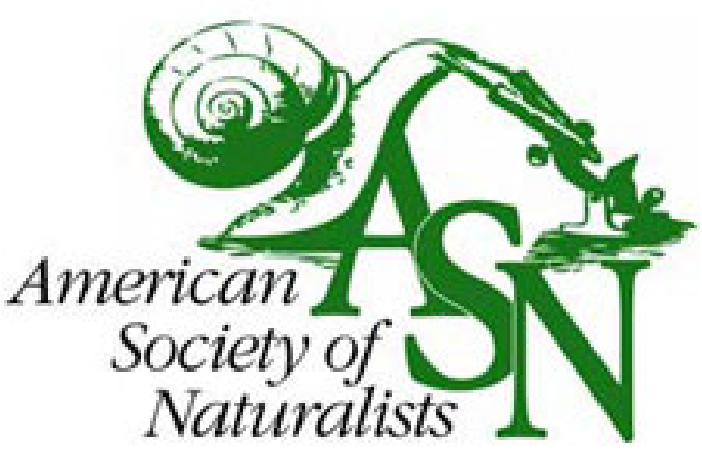

The University of Chicago

The Impact of Community Organization on Vector-Borne Pathogens.

Author(s): Benjamin Roche, Pejman Rohani, Andy P. Dobson, and Jean-François Guégan

Reviewed work(s):

Source: The American Naturalist, Vol. 181, No. 1 (January 2013), pp. 1-11

Published by: The University of Chicago Press for The American Society of Naturalists

Stable URL: http://www.jstor.org/stable/10.1086/668591

Accessed: 18/12/2012 13:20

Your use of the JSTOR archive indicates your acceptance of the Terms \& Conditions of Use, available at

http://www.jstor.org/page/info/about/policies/terms.jsp

JSTOR is a not-for-profit service that helps scholars, researchers, and students discover, use, and build upon a wide range of content in a trusted digital archive. We use information technology and tools to increase productivity and facilitate new forms of scholarship. For more information about JSTOR, please contact support@ jstor.org. 


\title{
The Impact of Community Organization on Vector-Borne Pathogens
}

\author{
Benjamin Roche, ${ }^{1,2,3 *}$ Pejman Rohani, ${ }^{2,4,5}$ Andy P. Dobson, ${ }^{6}$ and Jean-François Guégan ${ }^{3,7}$ \\ 1. Unité Mixte Internationale (UMI) Unité de Modélisation Mathématique et Informatique des Systèmes Complexes (UMMISCO) \\ IRD-UPMC 209, Centre IRD France Nord, 93143 Bondy, France; 2. Department of Ecology and Evolutionary Biology, University of \\ Michigan, Ann Arbor, Michigan 48109; 3. Unité Mixte de Recherche (UMR) Maladies Infectieuses et Vecteurs Écologie, Génétique, \\ Évolution, et Contrôle (MIVEGEC) IRD-CNRS-Universities of Montpellier I and II, Centre IRD de Montpellier, BP 64501, 34394 \\ Montpellier Cedex 5, France; 4. Center for the Study of Complex Systems, University of Michigan, Ann Arbor, Michigan 48109; \\ 5. Fogarty International Center, National Institutes of Health, Bethesda, Maryland 20892; 6. Department of Ecology and Evolutionary \\ Biology, University of Princeton, Princeton, New Jersey 08544; 7. Interdisciplinary Center on Biodiversity, Climate Change, and \\ Infectious Diseases, French School of Public Health, Montpellier, France
}

Submitted November 1, 2011; Accepted August 8, 2012; Electronically published November 27, 2012

Online enhancement: appendix PDF.

\begin{abstract}
Vector-borne zoonotic disease agents, which are known to often infect multiple species in the wild, have been identified as an emerging threat to human health. Understanding the ecology of these pathogens is especially timely, given the continued anthropogenic impacts on biodiversity. Here, we integrate empirical scaling laws from community ecology within a theoretical reservoir-vectorpathogen framework to study the transmission consequences of host community structure and diversity within large assemblages. We show that heterogeneity in susceptibility of the reservoir species promotes transmission "dilution," while a greater vector species richness "amplifies" it. These contrasting transmission impacts of vector and reservoir communities can yield very different epidemiological patterns. We demonstrate that vector and reservoir species richness can explain per se most of the pathogen transmission observed for West Nile virus in different parts of the United States, giving empirical support for the validity of these opposing theoretically predicted effects. We conclude that, in the context of disease emergence, the integration of a community perspective can provide critical insights into the understanding of pathogen transmission in wildlife.
\end{abstract}

Keywords: community epidemiology, emerging vector-borne diseases, community ecology, mathematical modeling.

\section{Introduction}

The recognition of zoonotic emerging infectious diseases (Daszak et al. 2001; Taylor et al. 2001) as a significant and permanent threat to human health has highlighted substantial gaps in our knowledge of infectious agents in their reservoir species, the structure and diversity of their host community networks, and the determinants of spillovers

\footnotetext{
* Corresponding author; e-mail: benjamin.roche@ird.fr.

Am. Nat. 2013. Vol. 181, pp. 1-11. (c) 2012 by The University of Chicago. 0003-0147/2013/18101-53428\$15.00. All rights reserved. DOI: $10.1086 / 668591$
}

enhancing concomitant human risk (Collinge and Ray 2006). This is particularly true of the potential threat posed by vector-borne diseases, which are transmitted between reservoir species via arthropod blood meals (Daszak et al. 2001; Morens et al. 2004; Gratz 2006). Yet our understanding of the epidemiology of vectored pathogens stems mainly from the substantial and important body of empirical and theoretical research that has focused on systems with simplified ecology-typically human diseases, such as malaria and dengue.

Improving our understanding of the ecosystem context of zoonoses is important because of long-standing concerns over anthropogenic effects on biodiversity (Millennium Ecosystem Assessment 2005; Keesing et al. 2010). We need to predict the transmission consequences of impoverished animal communities to prevent future epidemics arising from wildlife. In a set of pioneering empirical studies of Lyme disease, Ostfeld and colleagues showed that species-rich host reservoir communities are associated with lower pathogen prevalence (Ostfeld and Keesing 2000a, 2000b; LoGiudice et al. 2003, 2008; Keesing et al. 2010). This phenomenon, termed the "dilution effect" (Ostfeld and Keesing 2000a), results from a reduction in disease transmission as the relative abundance of reservoirs with low susceptibility increases (LoGiudice et al. 2003). While the empirical observation of the dilution effect has stimulated new research on how reservoir diversity influences infectious diseases dynamics (Ros and Pugliese 2007; Keesing et al. 2010), a mechanistic theoretical framework is currently lacking. One of the few theoretical treatments of this issue is from Dobson (2004), who assumed a simplified host community and examined the impact of host diversity on pathogen circulation with a frequency-depen- 
dent transmission. He confirmed that, when transmission is frequency dependent (Anderson and May 1991; McCallum et al. 2001), the inclusion of reservoir species with low susceptibility can dilute pathogen transmission. The extent to which these findings apply to more diverse and realistic host assemblages is unclear.

Here, we aim to fill this void by integrating community ecology and disease ecology in a mathematical framework. Our goal is to analyze vector-borne transmission dynamics within empirically motivated species assemblages representing both reservoir and vectors and the epidemiological consequences of their susceptibilities, abundances, and trophic preferences for vector species. Our analysis aims to understand how greater vector and/or reservoir species richness can impact pathogen transmission and whether there is some feedback between them. We then examine the veracity of these ideas via a case study, namely, the ecology of West Nile virus in different regions of the United States.

\section{A Community Epidemiology Framework for Vector-Borne Diseases}

Within the specific context of vector-borne diseases, there are two kinds of hosts: vectors and reservoir species. Thus, to avoid confusion we avoid the term "host" henceforth and instead define reservoir as a vertebrate host and vector as a biting insect. Within vectors and reservoirs, we assume infection dynamics to be well described by the susceptible, infectious, and recovered (SIR) model, in which individuals are categorized according to their infection status (Anderson and May 1991; Grenfell and Dobson 1995; Keeling and Rohani 2008). We present the extended multiple-species SIR model as a set of coupled differential equations:

$$
\begin{aligned}
\frac{d S_{i}}{d t} & =r_{i} N_{i}-\lambda_{i} S_{i}-\mu_{i} S_{i}, \\
\frac{d I_{i}}{d t} & =\lambda_{i} S_{i}-\left(\sigma_{i}+\mu_{i}\right) I_{i}, \\
\frac{d R_{i}}{d t} & =\sigma_{i} I_{i}-\mu_{i} R_{i},
\end{aligned}
$$

where $i$ is the species index for both vector and reservoir species (i.e., $i=1,2, \ldots, m+n$ ). For any species $i$, parameters $r_{i}$ and $\mu_{i}$ are the per capita birth and death rates, respectively; $N_{i}$ represents abundance, and $1 / \sigma_{i}$ denotes the mean infectious period. Note that in practice we assume infection dynamics in vectors to be given by an SI framework, such that $\sigma_{i}=0$ for $i=1, \ldots, m$. As we outline below, species-specific abundance $\left(N_{i}\right)$ and demographic rates $\left(\mu_{i}\right.$ and $r_{i}$ ) are quantified through empirical laws described in community ecology (described below). Pathogen transmission, assumed to be frequency dependent, is determined by the species-specific force of infection $\lambda_{i}$, which is given by:

$$
\lambda_{i}=\sum_{j=1}^{m+n} \phi_{i j} \tau_{i} \frac{I_{j}}{N_{j}} .
$$

Here, $\phi_{i j}$ quantifies the transmission rate from species $j$ to species $i$, is assumed to be constant in time, and is modulated by species-specific susceptibility, $\tau_{i}$. We assume that the abundances of vector community, composed of $m$ arthropod species, and those of the reservoir community, consisting of $n$ vertebrate species, are disconnected (fig. 1).

We assume the distribution of susceptibility within reservoir and vector communities is described by two independent truncated gamma distributions (defined by parameters $\left\{k_{\mathrm{V}}, \omega_{\mathrm{V}}\right\}$ and $\left\{k_{\mathrm{R}}, \omega_{\mathrm{R}}\right\}$ for vector and reservoir community, respectively). The gamma distribution is used to ensure a high flexibility of distribution shapes and is truncated to remain between 0 and 1 (fig. 1A). Susceptibility is assumed to be identical across all individuals within a given species.

\section{Integrating Lessons from Community Ecology}

Rather than attempting to explicitly model the population dynamics of each species, we take advantage of wellestablished empirical distributions associating species richness and species abundance (fig. $1 B$; Cody and Diamond 1975; May 1975). Specifically, we follow Fisher's classic result for invertebrate communities (Fisher et al. 1943) and assume vector abundances and vector species richness to be described by a geometric distribution:

$$
s_{v}(N)=\frac{\rho x^{N}}{N},
$$

where $s_{v}$ is the number of species with $N$ individuals, $x$ is a positive constant less than 1 , and $\rho$ an indicator of species diversity.

Similarly, we use Preston's law, giving a lognormal distribution, to describe the abundance of vertebrate reservoir species (Preston 1948; May 1975):

$$
s_{r}(P)=Y_{0} e^{-(z P)^{2}},
$$

where $z$ is a constant calculated from field data $(z<1$; see Preston 1948 for calculation), $s_{r}$ is the number of species falling into the $P$ th rank to the left or right of the mode, and $Y_{0}$ is the number of species in this modal rank.

It is important to point out that, using our framework, any increase or decrease in the species richness of vector or reservoir species will also result in a change in the total number of individuals in the community. Consequently, the epidemiological shifts following changes in vector and 


\section{Vector community} (species $[1, \mathrm{~m}])$

\section{Reservoir community $($ species $[m+1, m+n])$}
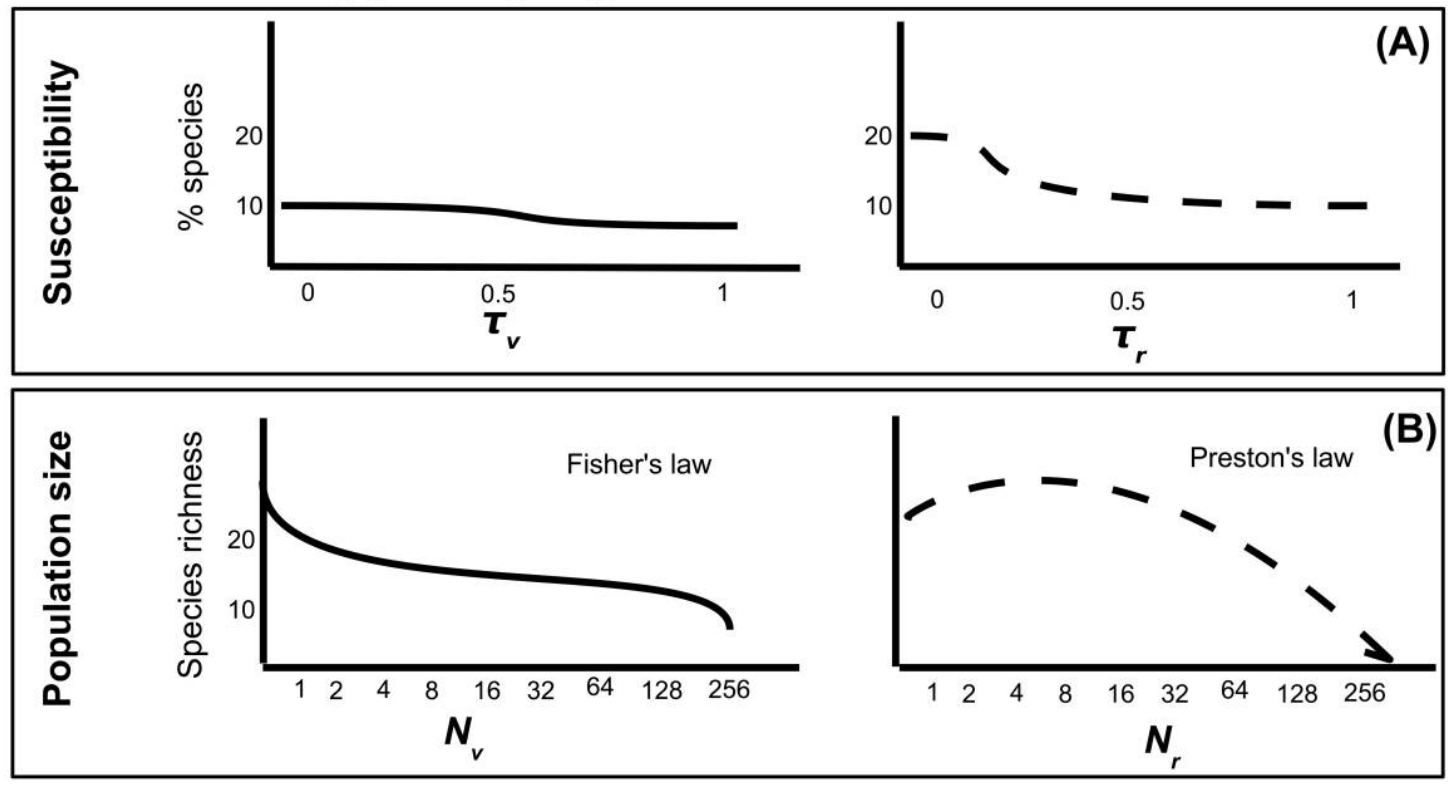

Figure 1: Summary of the community epidemiology framework. A, Susceptibilities of vector and reservoir species are modeled by two distinct gamma distributions ( $Y$-axis characterizes the proportion of species that have susceptibility quantified on $X$-axis). $B$, Characterization of the species abundance distribution. Fisher's law and Preston's law give the number of vector and reservoir species with a given abundance, respectively. We assume that the most abundant vector and reservoir species has the highest susceptibility and the fastest demographic turnover rate for reservoir species. Characteristics of vector and reservoir communities are assumed to be independent from each other.

reservoir species richness result from the combination of changing species richness and population abundance.

We distinguish between the demographic traits of different host species. Because of the relative taxonomic relatedness of vector species, their demographic rates are of the same order of magnitude (Spielman and D'Antonio 2001). Thus, for simplicity we assume that the demographic rates are identical for all vector species $\left(1 / r_{1 \leq i \leq m}=1 / \mu_{1 \leq i \leq m}=\right.$ 30 days; Spielman and D'Antonio 2001).

In contrast, we assume per capita birth and death rates for reservoir species to be determined by body mass via the following allometric relationship (Cohen et al. 2003):

$$
r_{i}=0.6 e^{a-b \cdot \log (i)^{-0.27}} .
$$

Here, the expression $e^{a-b \cdot \log (i)}$, derived from Jonnsson et al. (2005), represents the body mass of species $i$ according to its abundance rank, with $a$ and $b$ constant. The constant 0.6 has been estimated with field data (DeLeo and Dobson 1996). For the sake of simplicity, we assume here that birth and death rates are equal $\left(\mu_{i}=r_{i}\right)$, yielding constant population abundance.

Finally, we assume that (i) all reservoir species have the same infectious period $\left(1 / \sigma_{i}=7\right.$ days, for $m+1 \leq i \leq$ $m+n$ ), (ii) all vector species remain infectious until death $\left(\sigma_{i}=0\right.$, for $1 \leq i \leq m$; Spielman and D'Antonio 2001), and (iii) distribute bites uniformly on each reservoir species at a constant rate $\theta$. This parameter is included in matrix $\phi_{i j}$ (matrix characterization is given in section S1 of the appendix, available online).

\section{Simulation Protocol and Analysis}

Our aim is to quantify the influence of vector and reservoir species diversity (i.e., species richness and relative abundance) and composition (i.e., distribution of susceptibilities) on pathogen transmission. Ideally, an analytical treatment of this problem would shed light on epidemiologically important quantities, such as the basic reproduction ratio, $R_{0}$. However, for the complex and realistic scenarios we have in mind this is not possible, and we have instead adopted a simulation approach. An epidemiological metric that we use for quantification includes the maximal disease prevalence in vectors:

$$
I_{\max }=\max \frac{\sum_{k=1}^{m} I_{k}(t)}{\sum_{k=1}^{m} N_{k}},
$$


Table 1: Parameters used for the baseline scenario

\begin{tabular}{llc}
\hline Parameter & \multicolumn{1}{c}{ Biological meaning } & Value \\
\hline $\begin{array}{ll}\text { Vector community: } \\
x\end{array}$ & Constant used to characterize abundance distribution & .98 \\
$\rho$ & Diversity indicator used to characterize abundance distribution & 2 \\
$\mu_{1 \leq i \leq m}$ & Birth and death rates & 30 days $^{-1}$ \\
$\sigma_{1 \leq i \leq m}$ & Recovery rate of vector species & 0 \\
$1 / \theta$ & Period between two bites & 10 days \\
$k_{\mathrm{V}}$ & First parameter for gamma law characterizing susceptibilities & .02 \\
$\omega_{\mathrm{V}}$ & Second parameter for gamma law characterizing susceptibilities & 5 \\
Reservoir community: & & .1 \\
$z$ & Constant used to characterize abundance distribution & 4 \\
$P$ & Modal rank & 10 \\
$Y_{0}$ & Number of species in the modal rank & 7 days ${ }^{-1}$ \\
$\sigma_{m+1 \leq i \leq m+n}$ & Recovery rate & 0.7 \\
$a$ & First coefficient of allometric relationship characterizing demographic rates \\
$b$ & Second coefficient of allometric relationship characterizing demographic rates & 0 \\
$k_{\mathrm{R}}$ & First parameter for gamma law characterizing susceptibilities & .5 \\
$\omega_{\mathrm{R}}$ & Second parameter for gamma law characterizing susceptibilities & 1 \\
\hline
\end{tabular}

which is the proportion of infectious individuals across all vector species at the epidemic peak. This surrogate quantity is positively associated with $R_{0}$, although the relationship is nonlinear, as we show in section S2 of the appendix. Practically, $I_{\max }$ is informative about the risk of human exposure to a zoonotic pathogen since vectors represent the main source of infection for human populations. Finally, maximal disease prevalence in the vector community is generally used in field studies to evaluate the epidemiological activity of zoonotic vector-borne infections, which allows a direct comparison between outcomes of our model and published data.

To conduct our simulations (see section S3 of the appendix for algorithms), we take the following steps. First, we define the number of vector $(m)$ and reservoir $(n)$ species to create an array of species abundances $\left(N_{i}\right)$ of length $m+n$. Second, we generate an array abundance rank, ranging from 1 to $10^{5}$ on a $\log _{2}$ base. We then apply Fisher's law (eq. [5]) and Preston's law (eq. [6]), respectively, to calculate the number of vector and reservoir species within each population category, or octave. These values are then used to populate the array $N_{i}$. We then apply the allometric relationship described in equation (7) to determine reservoir birth and death rates of each species, assuming that the most abundant species has the fastest renewal rate (DeLeo and Dobson 1996). Next, we use the truncated gamma distribution to arrive at the distribution of species susceptibilities, assuming the most abundant species to have the highest susceptibility (consistent with empirical observations reported by Ostfeld and Keesing [2000a] and Keesing et al. [2006] as well as prior theoretical studies, as in Dobson [2004]). Finally, one infectious individual is introduced into the most susceptible vector species, and the SIR differential equations are applied.
All results presented below start with a baseline scenario in which parameters have been chosen in the region where a "dilution effect" (Ostfeld and Keesing 2000a) can occur in order to assess its generality. Specifically, the parameter set is intended to reflect West Nile virus epidemiology (table 1 provides these values). This baseline example allows us to start from a well-studied scenario involving a range of vector and reservoir species (McLean et al. 2001; Goddard et al. 2002; Granwehr et al. 2004; Reisen et al. 2004; Jourdain et al. 2007) and where a dilution effect has been empirically documented (Ezenwa et al. 2006). From this starting point, we can systematically modify vector and reservoir compositions and community structures (quantified respectively by their distribution of susceptibilities and species abundances) to explore their respective contributions to pathogen transmission. Then, we study the consequences of changing simultaneously the species richness of both vector and reservoir communities with respect to pathogen transmission. To focus exclusively on the effects of species richness, diversity, and composition, we set the parameter-defining variance of body mass distributions $(b)$ to 0 to have an identical demographic rate $(\mu)$ among reservoir species. Finally, it is assumed that all vectors are generalists. The influence of body mass distribution and vector trophic preferences are detailed in sections S4 and S5 of the appendix.

\section{Results}

\section{Influence of Community Composition: The Susceptibility} Distribution of Vector and Reservoir Species

The influence of vector and reservoir community composition is represented as a function of the mean and 
variance of their distributions of susceptibility (fig. 2). A clear message emerging from this analysis is that the average vector and reservoir susceptibility drives the intensity of disease transmission (fig. 2). Hence, for both communities, when a species is replaced by one that is more susceptible (resulting in a rise in mean susceptibility), overall transmission intensity increases.

The impact on transmission of variance in susceptibility is different for vector and reservoir species. As we show in figure $2 \mathrm{~A}$, variance in vector susceptibility does not affect maximal disease prevalence, while increasing variance in reservoir susceptibility leads to a decrease in transmission (fig. 2B). This is because higher variance in reservoir susceptibility translates into a larger number of low-susceptibility reservoir species that results in "wasted bites," yielding overall lower infection prevalence.

\section{Influence of Community Structure: The Species Richness/Abundance Relationship}

We analyzed the influence of vector and reservoir community structure on intensity of pathogen transmission. Since both Preston's law and Fisher's law associate species richness and abundances through two parameters, we use the Shannon index to summarize species diversity within each community. Specifically, we use $H=\sum_{i} q_{i} \cdot \log \left(q_{i}\right)$, where $q_{i}=N_{i} / \sum_{i} N_{i}$ (Shannon 1948).

First, we analyze the impact of vector community structure on pathogen transmission. We find that an increase in the vector Shannon index can lead to an increase in maximal disease prevalence (fig. $3 A$ ). In the framework of
Fisher's law, which is assumed here to characterize the vector community structure, a greater Shannon index indicates, on average, a greater total abundance. The ratio between total vector and total reservoir hosts, which is known to be an important driver for the level of disease transmission (Anderson and May 1991; Keeling and Rohani 2008), is hence increased, which in turn enhances disease transmission.

The structure of reservoir species community has a different impact on pathogen transmission intensity. When the average reservoir susceptibility is low or moderate (fig. $3 B$, black line), an increase in the Shannon index of reservoir communities dilutes maximal disease prevalence. This is because the number of weakly susceptible reservoir species rises. This process is similar to the increase in reservoir susceptibility variance. We emphasize that a high average reservoir susceptibility can potentially masquerade as a dilution effect. Indeed, this high average implies the presence of many reservoir species with high susceptibility, leading to a sporadic increase and decrease in pathogen transmission intensity along the Shannon index of reservoir species, despite an overall diminution of pathogen circulation (fig. $3 B$, gray and light gray lines).

\section{Feedback between Vector and Reservoir Communities: The Concept of Dilution Capacity}

In this section, we focus on the influence of changing simultaneously the species richness of both vector and reservoir communities (through parameters $\rho$ and $Y_{0}$, respectively). To this end, we introduce the measure of di-
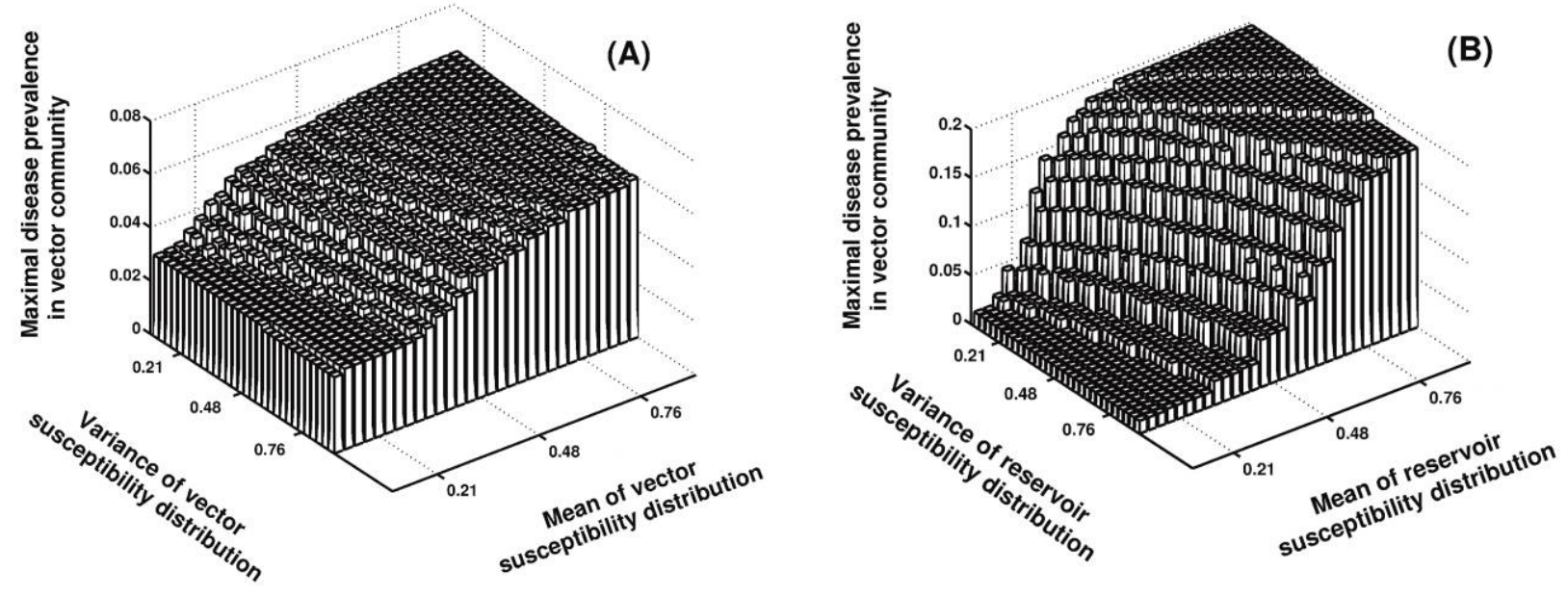

Figure 2: Relationship between distribution of species susceptibility and maximal disease prevalence. Maximal disease prevalence is defined as the maximal proportion of infectious vectors found in simulations (i.e., the epidemic peak). A, Influence of susceptibility distribution of vector species community. $B$, Influence of susceptibility distribution of reservoir species community. Parameter values are detailed in table 1 excepting $k_{\mathrm{R}}, \omega_{\mathrm{R}}, k_{\mathrm{V}}$, and $\omega_{\mathrm{V}}$, which are modified to explore different vector and reservoir community compositions. 

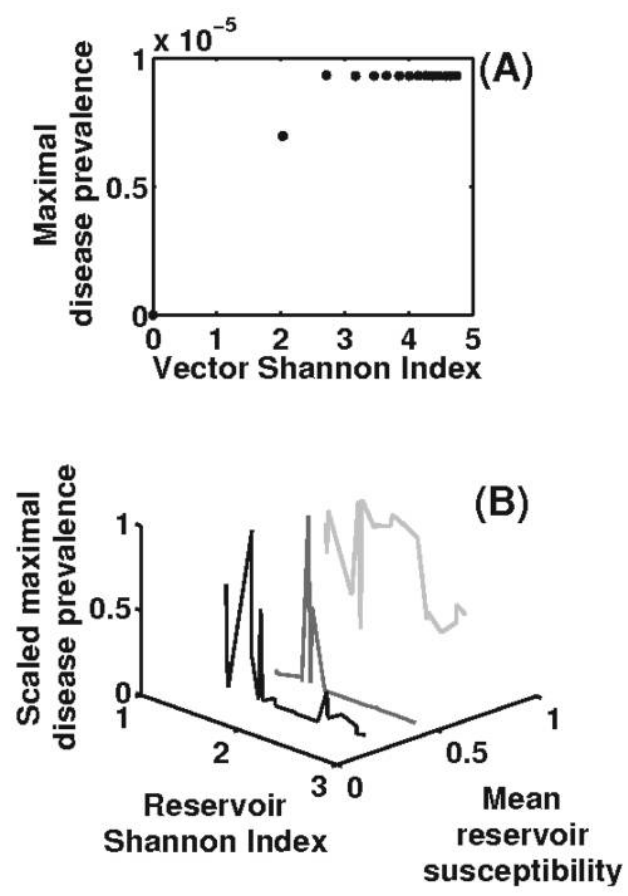

Figure 3: Relationship between species community characteristics and maximal disease prevalence. Maximal disease prevalence is defined as in figure 2. A, Influence of the Shannon index of the vector community on maximal disease prevalence. $B$, Relationship between the Shannon index of the reservoir community, its mean susceptibility, and maximal disease prevalence. Black, gray, and light gray lines represent mean reservoir susceptibility at $0.1,0.5$, and 0.9 , respectively. Parameter values are detailed in table 1 excepting $x$ and $\rho$, which are modified to explore vector species diversity, and $z$ and $Y_{0}$, which are modified to explore reservoir species diversity.

lution capacity. Let us consider two identical systems with identical community structures (for both vector and reservoir communities). A reservoir community composition can reach highly contrasting conclusions for any given mean susceptibility depending on the assumed variance in susceptibility. The dilution capacity is characterized by the ratio of maximal prevalences of vector communities when variance in susceptibility is either 0 or large, with all other parameters kept constant (fig. 4A). The dilution capacity highlights the maximal dilution effect that can be expected for a given structure of vector and reservoir communities.

Three important mechanisms can then be identified (fig. $4 B$ ). First, any increase in vector species richness is linked to a decrease in dilution capacity. An increase in vector species richness increases the total abundance of vector individuals and thus enhances the ratio between vector and reservoir individuals. Consequently, the increasing abundance of vector individuals of different species can compensate wasted bites for pathogens (bites on reservoir species that are weakly susceptible). Second, high vector species richness could be instrumental in triggering an epidemic because this increasing total vector abundance can be sufficient to overtake the threshold of pathogen invasion even if these additional vector species are weakly susceptible. Finally, an increase in reservoir species richness leads to an increase in dilution capacity because the reservoir community can accommodate more diversity in susceptibility and then offer more possibilities of wasted bites.

\section{Contrast with Empirical Data}

Our theoretical framework suggests that simultaneously changing vector and reservoir species richness can influence maximal disease prevalence in contrasting ways. Here, we examine the veracity of this result by confronting our theoretical framework with documented epidemiological situations observed for West Nile virus in two areas of the United States. West Nile virus disease is an emerging infectious disease caused by a flavivirus circulating in a variety of bird and mosquito species. This pathogen is highly suitable for our case study because of the large vector and reservoir spectra documented (McLean et al. 2001; Goddard et al. 2002; Granwehr et al. 2004; Reisen et al. 2004; Jourdain et al. 2007) and the long-lasting immunity generated in birds (Nemeth et al. 2009).

Susceptibility of 16 mosquito species (Goddard et al. 2002) and 25 bird species (Komar 2003) have been experimentally estimated. To ensure consistency, we define susceptibility as the infection probability after contact with an infectious individual (see "A Community Epidemiology Framework for Vector-Borne Diseases"), and we continue to assume, as explained above, that the most abundant species have the highest susceptibility. When more than 16 mosquito and 25 bird species are considered, we assume that the remaining species all have an identical susceptibility (quantified by a new parameter, $\xi$ ).

We describe the vector community with $x=0.998$, as is usually done for a mosquito community (Fisher et al. 1943). The reservoir community for West Nile virus is generally assumed to be composed mainly of bird species, especially passerine species (Balança and Hars 2004), and is accordingly described by $z=0.2$ (Preston 1948). We use variable values of $\rho$ and $Y_{0}$ (i.e., site-specific parameters of vector and reservoir communities, respectively) to explore a broad range of mosquito and bird species richness, respectively.

We assume that all mosquito species have a life span of 30 days and a biting rate of 1 bite every 10 days (Spielman and D'Antonio 2001). We characterize demographic rates of bird species using the same allometric relationship as described in "A Community Epidemiology Framework for Vector-Borne Diseases" (eq. [7]). We set the parameters 


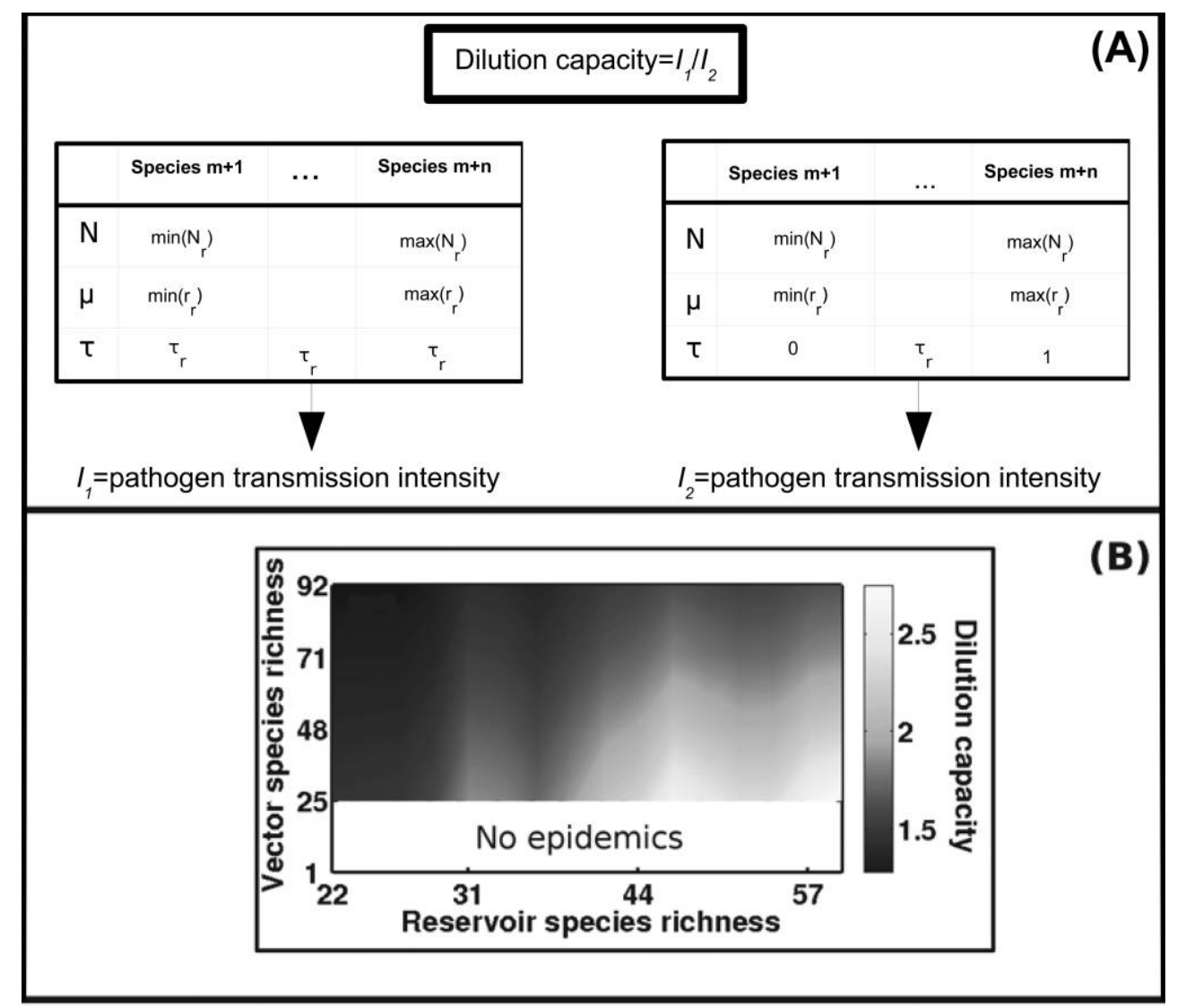

Figure 4: Influence of the feedback between reservoir and vector community richness on dilution capacity. A, Concept of dilution capacity. The strength of the dilution effect for a given average reservoir susceptibility $\left(\tau_{\mathrm{r}}\right)$ is characterized by the ratio of vector community maximal prevalences in two systems where variance in reservoir susceptibility is either $0\left(I_{1}\right)$ or large $\left(I_{2}\right)$, with all other parameters kept constant. $B$, Influence of reservoir and vector species richness on dilution capacity. A great dilution capacity describes a system where a strong dilution effect is expected (light area). A low dilution capacity (dark area) means that reservoir species richness is not expected to have a prophylactic effect on pathogen transmission. A dilution capacity equal to 0 (white area) represents situations in which epidemics cannot occur where variance in reservoir susceptibility distribution is maximal. Parameter values are detailed in table 1 excepting $\rho$ and $Y_{0}$, which are modified to explore vector and reservoir species diversity, respectively.

of this relationship according to a body mass distribution ranging from crows (up to $1.6 \mathrm{~kg}$ ) to the American robin (77 g; Svensson et al. 2010) because we assume that the reservoir community is composed essentially of passerine species (accordingly, $a=0.55$ and $b=0.76$ ).

We modify the parameters $\rho$ and $Y_{0}$ to explore the influence of vector and reservoir species richness on maximal pathogen prevalence. We then contrast maximal pathogen prevalence in the vector community predicted by our model against two empirical examples (fig. 5) from Louisiana (Ezenwa et al. 2006) and California (Reisen et al. 2004). In Louisiana, the observed prevalence in the vector community $\left(1.30 \times 10^{-3}\right.$; confidence interval: $4.82 \times 10^{-4}$ to $\left.3.80 \times 10^{-3}\right)$ is in moderate agreement with our model predictions (shaded in light gray in fig. 5) for similar values of mosquito and bird species richness sam- pled (seven mosquito and 25 bird species). Moving to the region of parameter space corresponding to California, which has greater mosquito and bird species richness (16 mosquito and 58 bird species), again we find reasonably strong agreement between the estimated prevalence $\left(3.28 \times 10^{-3}\right.$; confidence interval is unavailable) and model simulations (shaded in dark gray in fig. 5).

It is important to point out that congruence between estimated prevalence in California and our theoretical predictions depend on the susceptibility of bird species that have not been experimentally tested $(\xi)$. While an empirical estimate for this parameter is not available, we find the best match when $\xi$ is set to $4.7 \%$ (fig. 6). Below this value, the susceptibility of reservoir species is close to 0 , and the impact of the dilution effect is drastic. Above this value, there is no dilution effect. 


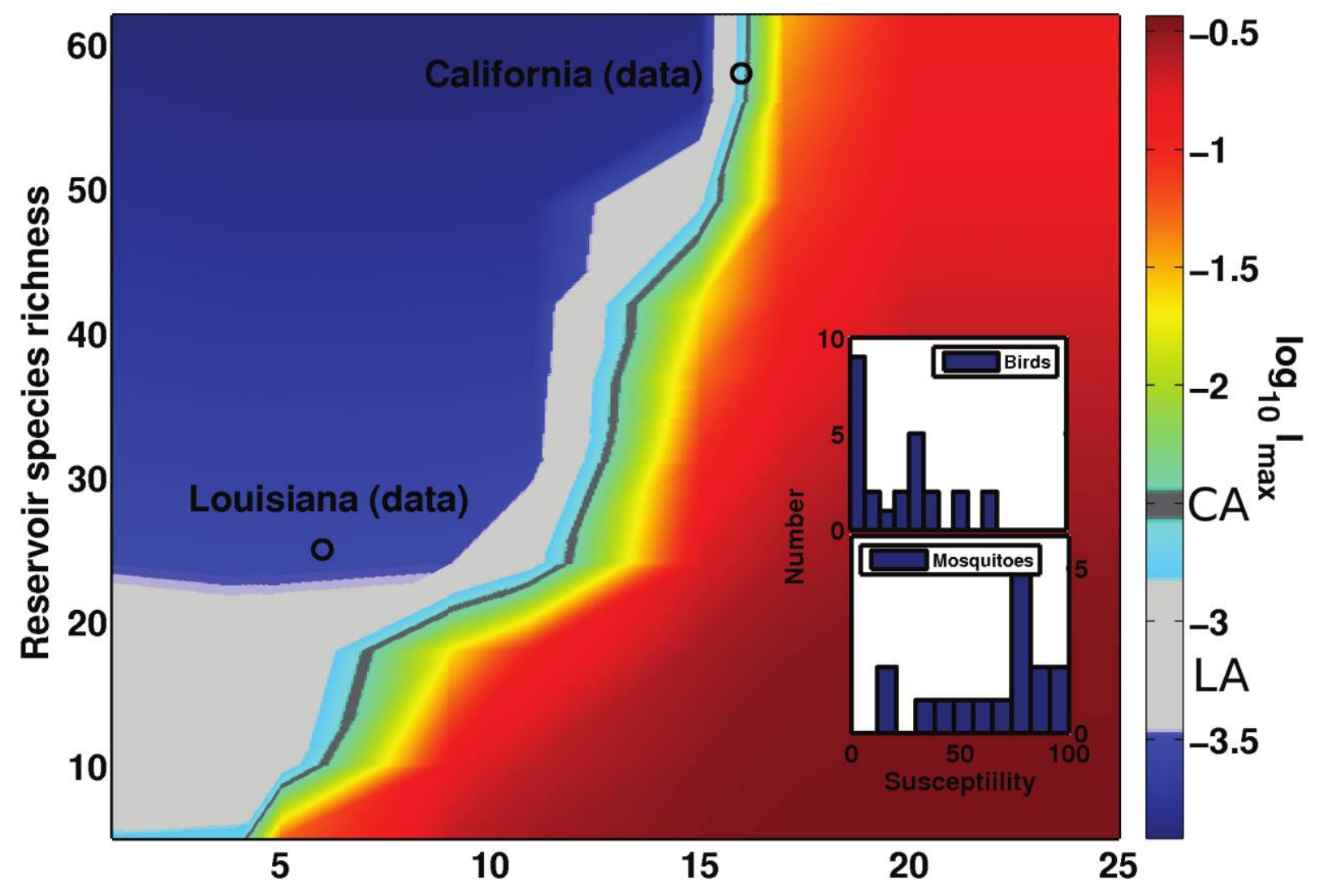

Vector species richness

Figure 5: Maximal disease prevalence generated by our theoretical framework $\left(\log _{10}\left(I_{\max }\right)\right)$ and their adequacy to real epidemiological situations (West Nile virus in Louisiana [LA] and California [CA]). The inset graph represents the susceptibility distribution of West Nile virus in mosquito and bird communities. Colors represent maximal disease prevalence generated by our model. The area shaded in light gray shows regions where the Louisiana prevalence is reproduced. The area shaded in dark gray shows regions where the California prevalence is reproduced. Circles show characteristics of the sampled communities within these locations. For visibility, we simulate more mosquito species than what have been experimentally tested by assuming that these mosquito species have a susceptibility of $12 \%$. Susceptibility of bird species not experimentally tested (parameter $\xi$ ) is set to $4.7 \%$ (see fig. 6). All parameters are detailed in the text.

\section{Discussion and Conclusion}

In this article, we have proposed a framework for the study of vector-borne diseases within species-rich networks of vector and reservoir communities. We have demonstrated that the protective effect of reservoir diversity on pathogen transmission is due to variance in reservoir susceptibility (fig. 2) rather than reservoir species richness per se. Particularly, we have shown that an increase in vector species richness may decrease the strength of any "dilution effect" such that it becomes negligible (fig. $4 B$ ). This is due to an increase in total vector abundance that enhances pathogen transmission (fig. $3 A$ ). Interestingly, we found that this increase in total vector abundance can also be crucial for epidemic takeoff, even if these new vector species are weakly susceptible. Finally, we propose than the interplay between vector and reservoir community richness can explain, at least in part, contrasting epidemiological observations of West Nile virus in the United States (fig. 5).

Previous theoretical studies have focused on small species assemblages (Dobson 2004) or on a specific disease (Foley et al. 2007; Ogden and Tsao 2009). By characterizing realistic and large vector and reservoir communities through the incorporation of empirical scaling laws from community ecology, our study extends these previous studies by testing the generality of their results, especially in the face of multiple vector species. We have demonstrated that vector species richness can impede the dilution effect and increase epidemic takeoff, even when these vector species are of low susceptibility. Our study has also shown that the dilution effect described for Lyme disease 


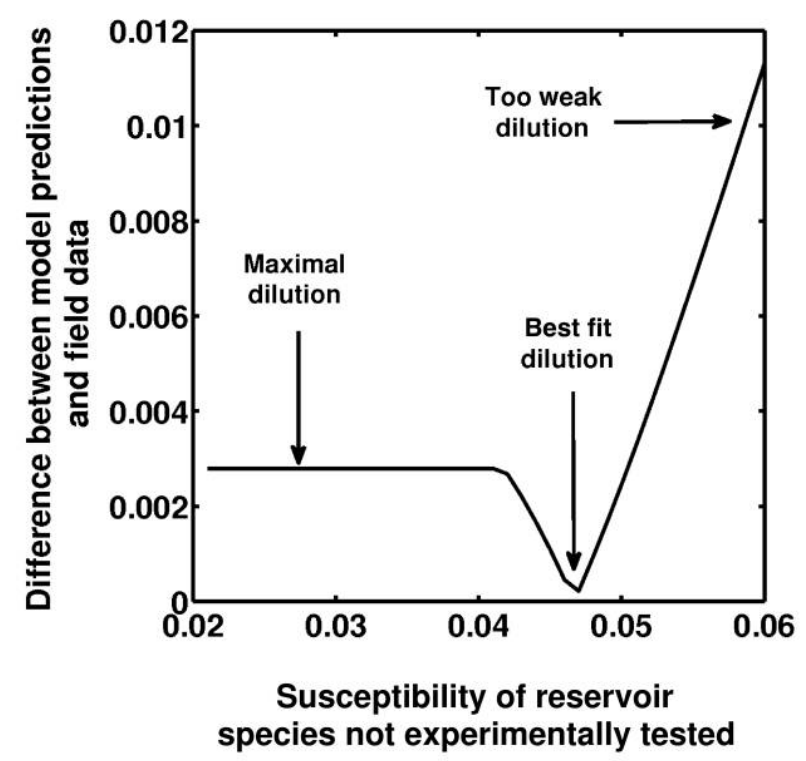

Figure 6: Adequacy between model predictions and data sampled in the field in California. The $Y$-axis shows the absolute value of the difference between the prevalence observed and that predicted in the whole vector community. The $X$-axis shows the susceptibility of reservoir species that have not been experimentally tested (parameter $\xi)$. The best-fit model assumes a reservoir susceptibility of $4.7 \%$.

(Ostfeld and Keesing 2000a) and West Nile virus (Ezenwa et al. 2006) may not be due to reservoir species richness per se but rather to variance in reservoir susceptibilities (fig. 2), as previously suggested in empirical studies (LoGiudice et al. 2003). Even if these two quantities are likely to be highly correlated in the field, the variance of reservoir susceptibility has to be cautiously considered.

We have analyzed the effects of species richness and susceptibility distribution for both vector and reservoir communities relative to an initial configuration exposed previously (see "A Community Epidemiology Framework for Vector-Borne Diseases"). Starting from a different configuration can contrast the role of species richness or susceptibility distribution. Nevertheless, our results are relevant for host-pathogen interactions that (i) are transmitted within species-rich communities where each species, reservoir, or vector has a different susceptibility and (ii) exhibit a lifelong immunity for reservoir species and no immunity in vector species. We believe that such systems are common and that our conclusions are valid for numerous cases.

The first hypothesis that deserved to be discussed is the independence between the characteristics of vector and reservoir communities. Our framework assumes that the abundances of vector and reservoir communities are disconnected, as is often assumed in the theoretical literature
(Anderson and May 1991; Keeling and Rohani 2008). It is plausible that reservoir abundance determines bloodfeeding opportunities for vectors and hence affects vector abundance. Consequently, an increase in reservoir abundance could also enhance vector abundance. Nevertheless, we submit that this assumption would lead only to a quantitative impact on our findings since it would solely increase the ratio of vector to reservoir abundance, leading to a reduced dilution effect.

The second hypothesis is the perfect correspondence between abundances and susceptibilities. We assume here that the most abundant vector and reservoir species are also the most susceptible. This assumption leads to an underestimation of the influence of weakly susceptible species, resulting in somewhat conservative conclusions. Extending the knowledge of the dilution effect requires this assumption because it is central in the dilution effect theory formulated by Ostfeld and colleagues (Schmidt and Ostfeld 2001). Our study adds realistic community structures and integrates the vector community; the natural next step for future work would be to relax this assumption.

Addressing a real case study through our modeling framework shows the limits of this perfect scaling between species abundance and susceptibility. This assumption has been included to keep continuity with previous studies of dilution effect theory (Schmidt and Ostfeld 2001), and it could be expected that such a relationship has exceptions, such as for the case of West Nile virus. Indeed, it has been shown that the American robin (Turdus migratorius), a bird species with a low abundance, is responsible for most West Nile virus transmission in the U.S. Northeast (Kilpatrick et al. 2006). Nevertheless, the confrontation of our theoretical predictions with empirical data is informative since our model fails to reproduce fairly the prevalence observed in Louisiana, where vector and reservoir species richness is low. In such a case, the importance of outliers along the relationship between susceptibility and abundance may drive the model to predict prevalences that are not perfectly consistent with observed data. This result emphasizes that community composition can play a strong role, especially when vector and reservoir species richness are low, as in Louisiana.

The case of species-rich communities in California shows that these exceptions may play a lesser role in such situations. Indeed, model-predicted West Nile virus prevalence in California is very close to the observations from field data. This better match between theory and empirical data is mainly due to the presence of weakly susceptible birds (with susceptibility estimated at $4.7 \%$ for an optimal match between model and data; see fig. 6). Then we demonstrate that the prevalence of West Nile virus observed in California can be reproduced for this value of the pa- 
rameter $\xi$. This result should be interpreted not as formal proof of a strong influence of these species in West Nile virus transmission, despite the suggestion made elsewhere (Ezenwa et al. 2006), but rather as a tentative explanation that deserved to be considered for West Nile virus. Addressing the relevance of such an explanation through quantifying susceptibility along a wider spectra of reservoir species to make comparisons with the parameter $\xi$ can be informative to address the potential generality of the dilution effect as well as to provide valuable insights into epidemics of West Nile virus and other multihost pathogens.

Our study has shown two main results. First, the dilution effect is produced by the diversity of reservoir susceptibility and not by reservoir species richness itself. From an evolutionary point of view, reservoir communities composed of species with very different susceptibilities can be supposed to have not experienced a long coevolutionary history with a pathogen except when the pathogen is an extreme generalist. It is therefore interesting that the dilution effect has been observed mainly in emerging diseases (Ostfeld and Keesing 2000b; Ezenwa et al. 2006), where this host-pathogen coevolutionary history is indeed absent. This result may highlight that the dilution effect is expected to be more frequent in emerging infections than for endemic pathogens. The second main result is the strong influence of vector species richness. Through their cumulative effect, these vector species, even with a low susceptibility, can be a determining factor in triggering epidemics or in buffering a potential dilution effect. More than the threat represented by the invasive nature of this group of species (Arim et al. 2006), identifying all the potential vectors of a given pathogen within an area is definitely a very complex issue, but it is a great challenge that has to be tackled to face future public health issues.

This study has extended disease transmission dynamics beyond the single host-single pathogen concept, aiming to embed this framework within the appropriate empirically motivated "community context." In addition to shedding light on well-studied empirical systems such as West Nile virus, this approach can inform preventive public health strategies regarding the impact of biodiversity alteration (Millennium Ecosystem Assessment 2005) on the emergence of zoonotic infectious diseases (Morens et al. 2004; Woolhouse and Gowtage-Sequeria 2005). The intuitive next steps would be to (i) relax the perfect scalin between abundance and susceptibility and (ii) integrate seasonality into the population dynamics of vector and vertebrate species. We submit that, when these steps are accomplished, a community epidemiology perspective can become crucial in anticipating and mitigating the threat of zoonotic pathogens.

\section{Acknowledgments}

We sincerely acknowledge the reviewers and the many colleagues who reviewed and improved the manuscript. In particular, we thank T. Ben Ari, M. Choisy, J. Drake, C. Lebarbenchon, K. Magori, and A. Park as well as the Rohani and King laboratories at the University of Michigan for their helpful comments. B.R. and J.-F.G. are sponsored by the Institut de Recherche pour le Développement, CNRS, and the French School of Public Health and acknowledge support from the EDEN (Emerging Diseases in a Changing European Environment) project (EU grant GOCE2003010284 EDEN). This publication is cataloged by the EDEN Steering Committee as EDEN089 (http:// www.eden-fp6project.net/). The contents of this publication are the sole responsibility of the authors and do not necessarily reflect the views of the European Commission. This work is a contribution from the DIVERSITASEcoHealth Alliance-ecoHEALTH research working group. J.-F.G. is supported by Laboratoire d'Excellence Centre d'Étude de la Biodiversité Amazonienne (grant ANR-10LABX-25-01). P.R. is supported by the Research and Policy in Infectious Disease Dynamics program of the Science and Technology Directorate, Department of Homeland Security, and by the Fogarty International Center, National Institutes of Health.

\section{Literature Cited}

Anderson, R. M., and R. M. May. 1991. Infectious diseases of humans: dynamics and control. Oxford Science, Oxford.

Arim, M., S. R. Abades, P. E. Neill, M. Lima, and P. A. Marquet. 2006. Spread dynamics of invasive species. Proceedings of the National Academy of Sciences of the USA 103:374-378.

Balança, G., and J. Hars. 2004. Bird reservoirs and indicators of the West Nile fever in France. Game and Wildlife Science 21:539-551.

Cody, M. L., and J. M. Diamond, eds. 1975. Ecology and evolution of communities. Harvard University Press, Cambridge, MA.

Cohen, J. E., T. Jonsson, and S. R. Carpenter. 2003. Ecological community description using the food web, species abundance, and body size. Proceedings of the National Academy of Sciences of the USA 100:1781-1786.

Collinge, S. H., and C. Ray, eds. 2006. Disease ecology: community structure and pathogen dynamics. Oxford University Press, New York.

Daszak, P., A. A. Cunningham, and A. D. Hyatt. 2001. Anthropogenic environmental change and the emergence of infectious diseases in wildlife. Acta Tropica 78:103-116.

DeLeo, G. A., and A. P. Dobson. 1996. Allometry and simple epidemic models for microparasites. Nature 379:720-722.

Dobson, A. 2004. Population dynamics of pathogens with multiple host species. American Naturalist 164(suppl.):S64-S78.

Ezenwa, V. O., M. S. Godsey, R. J. King, and S. C. Guptill. 2006. Avian diversity and West Nile virus: testing associations between biodiversity and infectious disease risk. Proceedings of the Royal Society B: Biological Sciences 273:109-117. 
$\rightarrow$ Fisher, R. A., A. S. Corbet, and C. B. Williams. 1943. The relation between the number of species and the number of individuals in a random sample of an animal population. Journal of Anima Ecology 12:42-58.

Foley, J. E., J. Zipser, B. Chomel, E. Girvetz, and P. Foley. 2007. Modeling plague persistence in host-vector communities in Cal $\rightarrow$ ifornia. Journal of Wildlife Diseases 43:408-424.

$\rightarrow$ Goddard, L. B., A. E. Roth, W. K. Reisen, and T. W. Scott. 2002. Vector competence of California mosquitoes for West Nile virus. Emerging Infectious Diseases 8:1385-1391.

$\rightarrow$ Granwehr, B. P., K. M. Lillibridge, S. Higgs, P. W. Mason, J. F. Aronson, G. A. Campbell, and A. D. T. Barrett. 2004. West Nile virus: where are we now? Lancet Infectious Diseases 4:547-556.

Gratz, N. 2006. Vector- and rodent-borne diseases in Europe and North America: distribution, public health burden and control. Cambridge University Press, Cambridge.

Grenfell, B. T., and A. P. Dobson, eds. 1995. Ecology of infectious diseases in natural populations. Cambridge University Press, Cambridge.

$\rightarrow$ Jonnsson, T., J. E. Cohen, and S. R. Carpenter. 2005. Food webs, body size and species abundance in ecological community description. Advances in Ecological Research 36:1-84.

$\rightarrow$ Jourdain, E., Y. Toussaint, A. Leblond, D. J. Bicout, P. Sabatier, and M. Gauthier-Clerc. 2007. Bird species potentially involved in introduction, amplification and spread of West Nile virus in a Mediterranean wetland, the Camargue (southern France). VectorBorne and Zoonotic Diseases 7:15-33.

Keeling, M. J., and P. Rohani. 2008. Modeling infectious diseases in humans and animals. Princeton University Press, Princeton, NJ.

$\rightarrow$ Keesing, F., L. K. Belden, P. Daszak, A. Dobson, C. D. Harvell, R. D. Holt, P. Hudson, et al. 2010. Impacts of biodiversity on the emergence and transmission of infectious diseases. Nature 468:647652.

$\rightarrow$ Keesing, F., R. Holt, and R. Ostfeld. 2006. Effects of species diversity on disease risk. Ecology Letters 9:485-498.

$\rightarrow$ Kilpatrick, A. M., P. Daszak, M. J. Jones, P. P. Marra, and L. D. Kramer. 2006. Host heterogeneity dominates West Nile virus transmission. Proceedings of the Royal Society B: Biological Sciences 273:2327-2333.

$\rightarrow$ Komar, N. 2003. West Nile virus: epidemiology and ecology in North America. Advances in Virus Research 61:185-234.

$\rightarrow$ LoGiudice, K., S. T. K. Duerr, M. J. Newhouse, K. A. Schmidt, M E. Killilea, and R. S. Ostfeld. 2008. Impact of host community composition on Lyme disease risk. Ecology 89:2841-2849.

$\rightarrow$ LoGiudice, K., R. S. Ostfeld, K. A. Schmidt, and F. Keesing. 2003 The ecology of infectious disease: effects of host diversity and community composition on Lyme disease risk. Proceedings of the National Academy of Sciences of the USA 100:567-571.

May, R. M. 1975. Patterns of species abundance and diversity. Pages 81-120 in M. L. Cody and J. M. Diamonds. Ecology and evolution of communities. Chapter 4. Harvard University Press, Cambridge, MA.

McCallum, H., N. Barlow, and J. Hone. 2001. How should pathogen transmission be modelled? Trends in Ecology \& Evolution 16:295300.

McLean, R. G., S. R. Ubico, D. E. Docherty, W. R. Hansen, L. Sileo, and T. S. McNamara. 2001. West Nile virus transmission and ecology in birds. Annals of the New York Academy of Sciences 951:54-57.

Millennium Ecosystem Assessment. 2005. Ecosystem and human well-being: biodiversity synthesis. World Resources Institute, Washington, DC.

Morens, D. M., G. K. Folkers, and A. S. Fauci. 2004. The challenge of emerging and re-emerging infectious diseases. Nature 430:242249.

Nemeth, N. M., P. T. Oesterle, and R. A. Bowen. 2009. Humoral immunity to West Nile virus is long-lasting and protective in the house sparrow (Passer domesticus). American Journal of Tropical Medicine and Hygiene 80:864-869.

Ogden, N. H., and J. I. Tsao. 2009. Biodiversity and Lyme disease: dilution or amplification? Epidemics 1:196-206.

Ostfeld, R. S., and F. Keesing. 2000a. Biodiversity and disease risk: the case of Lyme disease. Conservation Biology 14:722-728.

. 2000 $b$. The function of biodiversity in the ecology of vectorborne zoonotic diseases. Canadian Journal of Zoology 78:20612078.

Preston, F. W. 1948. The commonness, and rarity, of species. Ecology 29:254-283.

Reisen, W., H. Lothrop, R. Chiles, M. Madon, C. Cossen, L. Woods, S. Husted, V. Kramer, and J. Edman. 2004. West Nile virus in California. Emerging Infectious Diseases 10:1369-1378.

Ros, R., and A. Pugliese. 2007. Effects of tick population dynamics and host densities on the persistence of tick-borne infections. Math Biosciences 208:216-240.

Schmidt, K. A., and R. S. Ostfeld. 2001. Biodiversity and the dilution effect in disease ecology. Ecology 3:609-619.

Shannon, C. E. 1948. A mathematical theory of communication. Bell System Technical Journal 27:379-423.

Spielman, A., and M. D’Antonio. 2001. Mosquito: a natural history of our most persistent and deadly foe. Hyperion, New York.

Svensson, L., D. Zetterstrom, and K. Mullarney, eds. 2010. Birds of Europe. Princeton University Press, Princeton, NJ.

$\rightarrow$ Taylor, L. H., S. M. Latham, and M. E. Woolhouse. 2001. Risk factors for human disease emergence. Philosophical Transactions of the Royal Society B: Biological Sciences 356:983-989.

$\rightarrow$ Woolhouse, M. E. J., and S. Gowtage-Sequeria. 2005. Host range and emerging and reemerging pathogens. Emerging Infectious Diseases 11:1842-1847.

Associate Editor: Marc Mangel Editor: Mark A. McPeek 